\title{
Cryogenic Behavior of Selected Magnetic Materials
}

\author{
J. J. Gniewek and E. Ploge* \\ Institute for Materials Research, National Bureau of Standards, Boulder, Colo.
}

(April 22, 1965)

\begin{abstract}
Measurements of a-c core loss and d-c magnetic properties including coercive force, residual induction, and hysteresis loss are reported at temperatures of $273,195,76$, and $4{ }^{\circ} \mathrm{K}$ for a variety of commercial magnetic Fe-Si and Fe-48 percent $\mathrm{Ni}$ alloys. Of the materials tested the high silicon content ( 3 to 4.45 percent $\mathrm{Si}$ ) $\mathrm{Fe}-\mathrm{Si}$ alloys were found to be the least temperature sensitive. Hysteresis loss increases between 273 and $4{ }^{\circ} \mathrm{K}$ of up to 100 percent were measured for the iron-nickel materials.
\end{abstract}

\section{Introduction}

An increasing use of electric motors, transformers, and other electrical equipment operating at cryogenic temperatures has created the need for engineering data on the low-temperature magnetic properties of ferromagnetic materials. To fill this need, a-c and d-c magnetic properties were measured at $4,76,195$, and $273^{\circ} \mathrm{K}$ for a variety of commercial $\mathrm{Fe}-\mathrm{Si}$ and $\mathrm{Fe}-\mathrm{Ni}$ alloys.

*Present address: Colorado Earth Science Laboratories, Inc., Boulder, Colo.

\section{Materials Tested}

Tables 1 and 2 list the various $\mathrm{Fe}-\mathrm{Si}$ and $\mathrm{Fe}-\mathrm{Ni}$ samples tested together with related pertinent information concerning sheet thickness, type of core, and chemical composition. The identification numbers given will be referred to throughout the text. Materials 110 through 118 were kindly supplied by United States Steel Corporation and materials 100 through 102 and 200 through 213 by Armco Steel Corporation. Materials 100 through 102 are identified by the trade name, "Oriented T," 200 through 203 by "48 Orthonik," and 210 through 213 by "48 Ni."

TABLE 1. Iron-silicon alloys tested at cryogenic temperatures

\begin{tabular}{|c|c|c|c|c|c|c|c|c|}
\hline \multirow{2}{*}{$\begin{array}{l}\text { Material AISI } \\
\text { Number }\end{array}$} & \multirow{2}{*}{$\begin{array}{l}\text { Type } \\
\text { product }\end{array}$} & \multicolumn{3}{|c|}{$\begin{array}{l}\text { Composition, percent } \\
\text { (balance primarily iron) }\end{array}$} & \multirow{2}{*}{ Grain orientation } & \multirow[t]{2}{*}{ Thickness } & \multirow[t]{2}{*}{ Type of core } & \multirow{2}{*}{$\begin{array}{l}\text { Identification } \\
\text { Number }\end{array}$} \\
\hline & & $\mathrm{Si}$ & $\mathrm{C}$ & $\mathrm{Mn}$ & & & & \\
\hline $\begin{array}{l}\text { Thin grain-oriented } \\
\text { nominally } 3 \text { percer } \\
\text { Si alloy. } \\
\text { M-43 } \\
\text { M-43 } \\
\text { M-36 } \\
\text { M-27 } \\
\text { M-27 } \\
\text { M-27 } \\
\text { M-22 } \\
\text { M-19 } \\
\text { M-15 }\end{array}$ & $\begin{array}{l}\text { nt Fe- } \\
\text { HR } \\
\text { HR } \\
\text { CR } \\
\text { CR } \\
\text { HR } \\
\text { HR } \\
\text { HR } \\
\text { HR } \\
\text { HR }\end{array}$ & $\begin{array}{l}2.78 \\
1.01 \\
0.85 \\
1.90 \\
2.93 \\
3.01 \\
2.93 \\
3.13 \\
3.57 \\
4.45\end{array}$ & $\begin{array}{l}0.03 \\
.03 \\
.05 \\
.04 \\
.04 \\
.017 \\
.04 \\
.03 \\
.021 \\
.043\end{array}$ & $\begin{array}{r}0.08 \\
.23 \\
.35 \\
.31 \\
.25 \\
.10 \\
.37 \\
.11 \\
.11 \\
.14\end{array}$ & 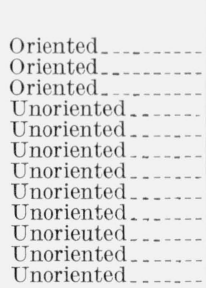 & $\begin{array}{l}\text { Inches } \\
0.001 \\
.002 \\
.004 \\
.0250 \\
.0185 \\
.0250 \\
.0250 \\
.0185 \\
.0250 \\
.0140 \\
.0140 \\
.0185\end{array}$ & $\begin{array}{l}\text { Tape-wound, B* } \\
\text { Tape-wound, B } \\
\text { Tape-wound, B } \\
\text { Laminated-U' } \\
\text { Laminated-U, B } \\
\text { Laminated-U, B } \\
\text { Laminated-U, } \\
\text { Laminated-U, B } \\
\text { Laminated-U } \\
\text { Laminated-U } \\
\text { Laminated-U, B } \\
\text { Laminated-B }\end{array}$ & $\begin{array}{l}100 \\
101 \\
102 \\
110 \\
111 \\
112 \\
113 \\
114 \\
115 \\
116 \\
117 \\
118\end{array}$ \\
\hline
\end{tabular}

*=boxed. $\quad{ }^{* *} \mathrm{U}=$ unboxed. $\quad \mathrm{HR}=$ hot rolled. $\quad \mathrm{CR}=$ cold rolled.

TABLE 2. Iron-nickel alloys tested at cryogenic temperatures Nominally 48 percent Ni content.

\begin{tabular}{|c|c|c|c|c|}
\hline Material & $\begin{array}{l}\text { Grain } \\
\text { orienta- } \\
\text { tion }\end{array}$ & Thickness & Type of core & $\begin{array}{l}\text { Identifica- } \\
\text { tion number }\end{array}$ \\
\hline $\begin{array}{l}\text { FeNi } \\
\text { FeNi } \\
\text { FeNi } \\
\text { FeNi } \\
\text { FeNi } \\
\text { FeNi } \\
\text { FeNi }\end{array}$ & $\begin{array}{l}\text { Oriented.-- } \\
\text { Oriented.-- } \\
\text { Oriented.-- } \\
\text { Oriented. } \\
\text { Unoriented } \\
\text { Unoriented } \\
\text { Unoriented } \\
\text { Unoriented. }\end{array}$ & $\begin{array}{l}\text { Inches } \\
0.002 \\
.004 \\
.006 \\
.006 \\
.002 \\
.004 \\
.006 \\
.014\end{array}$ & $\begin{array}{l}\text { Tape-wound, B } \\
\text { Tape-wound, B } \\
\text { Tape-wound, B } \\
\text { Laminated, B } \\
\text { Tape-wound, B } \\
\text { Tape-wound, B } \\
\text { Laminated, B } \\
\text { Laminated, B }\end{array}$ & $\begin{array}{l}200 \\
201 \\
202 \\
203 \\
210 \\
211 \\
212 \\
213\end{array}$ \\
\hline
\end{tabular}

\section{Measuring Technique}

\subsection{Equipment}

A standard voltmeter-wattmeter method $[1]^{1}$ was used to determine a-c core loss values. The magnetic induction in the core is determined by measuring the secondary voltage with an a-c differential voltmeter calibrated to 0.5 percent accuracy. Induction levels

1 Figures in brackets indicate the literature references at the end of this paper. 
were limited to values below that which resulted in 3 percent or greater 3rd harmonic distortion as determined by a wave analyzer across the secondary windings. A high input impedence electronic wattmeter was employed thereby making instrumental loss corrections negligible. Direct current hysteresis loops were plotted semiautomatically on an $x-y$ chart recorder. The magnetic induction was recorded using an integrating d-c amplifier. The magnetizing force was calculated from the voltage drop produced by the magnetizing current flowing through a standard resistor.

\subsection{Samples}

The samples used for cryogenic testing weigh approximately $1 / 8 \mathrm{lb}$ and are considerably smaller than that specified by the ASTM standards [2]. This was necessary to prevent excessive liquid evaporation and the use of inconveniently large Dewars. All samples are in the shape of toroids. The tape-wound and laminated samples vary slightly in size. In all cases, however, the ratio of mean diameter to radial width is 8 to 1 or greater. This ensures a reasonably uniform flux distribution throughout the cross-sectional area of the sample.
The mean diameter of the cores is approximately 2 in. For most materials two samples of each were tested.

The oriented iron-silicon material and all ironnickel samples were placed in either anodized aluminum or bakelite boxes prior to winding the secondary and primary coils. These loose-fitting boxes prevent coil winding stresses from affecting the magnetic properties. Since the unoriented grades of Fe-Si are seldom boxed in normal use, these samples were prepared by wrapping the laminated toroid with 0.004-in. Teflon tape to provide electrical insulation between windings and core material. The primary and secondary windings were then placed directly atop the Teflon tape. To determine the effect of winding stresses on the magnetic properties, four materials, $111,112,114$, and 117 , were also tested in boxed form. The large increase in core loss for the unboxed cores compared to the boxed cores at low temperature can be attributed to the detrimental stress effects produced by the differential contraction between the iron-core material and the copper windings. Because of the wide use of the unoriented grade $\mathrm{Fe}-\mathrm{Si}$ in components where little or no emphasis is given to eliminating winding or clamping stresses, core loss data for the unboxed as well as selected boxed materials are shown in table 3 .

TABLE 3. Total core loss at $273^{\circ} \mathrm{K}$ and the ratio of core loss measured at temperature $T$ to that measured at $T=273{ }^{\circ} \mathrm{K}$

(Core loss value at $273{ }^{\circ} \mathrm{K}$ is given in units of watts/pound)

\begin{tabular}{|c|c|c|c|c|c|c|c|c|c|c|c|}
\hline \multirow{2}{*}{ Material } & \multirow{2}{*}{ Frequency } & \multirow{2}{*}{ Temperature } & \multicolumn{9}{|c|}{ Peak induction $k G$} \\
\hline & & & 2 & 4 & 8 & 10 & 13 & 15 & 16 & 17 & 18 \\
\hline $100 \ldots$ & $\begin{array}{l}c / s \\
60\end{array}$ & $\begin{array}{r}{ }^{\circ} K \\
273 \\
195 \\
76 \\
4\end{array}$ & $\begin{array}{l}0.031 \\
1.03 \\
1.10 \\
1.13\end{array}$ & $\begin{array}{l}0.129 \\
1.02 \\
1.05 \\
1.08\end{array}$ & $\begin{array}{l}0.46 \\
1.02 \\
1.04 \\
1.07\end{array}$ & $\begin{array}{l}0.73 \\
1.01 \\
1.01 \\
1.03\end{array}$ & $\begin{array}{l}1.4_{0} \\
1.00 \\
1.00 \\
1.00\end{array}$ & & & & \\
\hline $100 \ldots$ & 400 & $\begin{array}{r}273 \\
195 \\
76 \\
4\end{array}$ & $\begin{array}{l}0.23_{0} \\
1.02 \\
1.07 \\
1.09\end{array}$ & $\begin{array}{l}0.91 \\
1.02 \\
1.05 \\
1.08\end{array}$ & $\begin{array}{l}3.1 \\
1.03 \\
1.06 \\
1.10\end{array}$ & $\begin{array}{l}5.0 \\
1.02 \\
1.06 \\
1.08\end{array}$ & $\begin{array}{l}9.4 \\
1.02 \\
1.04 \\
1.06\end{array}$ & $\begin{array}{c}15.1 \\
1.01 \\
1.02 \\
1.03\end{array}$ & & & \\
\hline $100 \ldots$ & 1000 & $\begin{array}{r}273 \\
195 \\
76 \\
4\end{array}$ & $\begin{array}{l}0.60 \\
1.03 \\
1.10 \\
1.13\end{array}$ & $\begin{array}{l}2.40 \\
1.03 \\
1.08 \\
1.12\end{array}$ & $\begin{array}{l}8.7 \\
1.01 \\
1.06 \\
1.08\end{array}$ & $\begin{array}{c}13.3 \\
1.02 \\
1.05 \\
1.07\end{array}$ & & & & & \\
\hline $101 \ldots$ & 60 & $\begin{array}{r}273 \\
195 \\
76 \\
4\end{array}$ & $\begin{array}{l}0.017_{8} \\
1.03 \\
1.07 \\
1.09\end{array}$ & $\begin{array}{l}0.070 \\
1.03 \\
1.06 \\
1.09\end{array}$ & $\begin{array}{l}0.239 \\
1.04 \\
1.07 \\
1.08\end{array}$ & $\begin{array}{l}0.36 \\
1.03 \\
1.06 \\
1.06\end{array}$ & $\begin{array}{l}0.64 \\
1.03 \\
1.06 \\
1.06\end{array}$ & $\begin{array}{l}1.03 \\
1.03 \\
1.05 \\
1.05\end{array}$ & & & \\
\hline $101 \ldots$ & 400 & $\begin{array}{r}273 \\
195 \\
76 \\
4\end{array}$ & $\begin{array}{l}0.137 \\
1.06 \\
1.13 \\
1.15\end{array}$ & $\begin{array}{l}0.54 \\
1.06 \\
1.13 \\
1.15\end{array}$ & $\begin{array}{l}1.84 \\
1.07 \\
1.13 \\
1.15\end{array}$ & $\begin{array}{l}2.9_{1} \\
1.03 \\
1.10 \\
1.10\end{array}$ & $\begin{array}{l}5.0 \\
1.02 \\
1.06 \\
1.08\end{array}$ & $\begin{array}{l}7.8 \\
1.03 \\
1.06 \\
1.09\end{array}$ & & $\begin{array}{c}14.0 \\
1.00 \\
1.00 \\
1.00\end{array}$ & \\
\hline $101 \ldots$ & 1000 & $\begin{array}{r}273 \\
195 \\
76 \\
4\end{array}$ & $\begin{array}{l}0.40 \\
1.08 \\
1.15 \\
1.18\end{array}$ & $\begin{array}{l}1.57 \\
1.06 \\
1.12 \\
1.13\end{array}$ & $\begin{array}{l}5.7 \\
1.07 \\
1.12 \\
1.12\end{array}$ & $\begin{array}{l}8.7 \\
1.07 \\
1.11 \\
1.13\end{array}$ & & & & & \\
\hline $102 \ldots$ & 60 & $\begin{array}{r}273 \\
195 \\
76 \\
4\end{array}$ & $\begin{array}{l}0.0127 \\
1.02 \\
1.06 \\
1.08\end{array}$ & $\begin{array}{l}0.046 \\
1.02 \\
1.04 \\
1.07\end{array}$ & $\begin{array}{l}0.15_{1} \\
1.04 \\
1.07 \\
1.09\end{array}$ & $\begin{array}{l}0.22_{0} \\
1.04 \\
1.08 \\
1.10\end{array}$ & $\begin{array}{l}0.37 \\
1.03 \\
1.08 \\
1.08\end{array}$ & $\begin{array}{l}0.53 \\
1.02 \\
1.06 \\
1.08\end{array}$ & $\begin{array}{l}0.73 \\
1.01 \\
1.01 \\
1.03\end{array}$ & & \\
\hline $102 \ldots$ & 400 & $\begin{array}{r}273 \\
195 \\
76 \\
4\end{array}$ & $\begin{array}{l}0.128 \\
1.09 \\
1.17 \\
1.20\end{array}$ & $\begin{array}{l}0.46 \\
1.09 \\
1.17 \\
1.22\end{array}$ & $\begin{array}{l}1.6_{1} \\
1.07 \\
1.16 \\
1.17\end{array}$ & $\begin{array}{l}2.38 \\
1.08 \\
1.16 \\
1.18\end{array}$ & $\begin{array}{l}3.9 \\
1.08 \\
1.18 \\
1.21\end{array}$ & $\begin{array}{l}5.5 \\
1.05 \\
1.09 \\
1.11\end{array}$ & $\begin{array}{l}7.1 \\
1.03 \\
1.06 \\
1.08\end{array}$ & & $\begin{array}{c}13.7 \\
1.01 \\
1.06 \\
1.09\end{array}$ \\
\hline $102 \ldots \ldots$ & 1000 & $\begin{array}{r}273 \\
195 \\
76 \\
4\end{array}$ & $\begin{array}{l}0.46 \\
1.07 \\
1.17 \\
1.20\end{array}$ & $\begin{array}{l}1.7_{4} \\
1.07 \\
1.16 \\
1.18\end{array}$ & $\begin{array}{l}6.2 \\
1.06 \\
1.16 \\
1.19\end{array}$ & $\begin{array}{l}9.4 \\
1.06 \\
1.16 \\
1.19\end{array}$ & & & & & \\
\hline
\end{tabular}




\begin{tabular}{|c|c|c|c|c|c|c|c|c|c|}
\hline \multirow{2}{*}{ Material } & \multirow{2}{*}{$\begin{array}{l}\text { Fre- } \\
\text { quen- } \\
\text { cy }\end{array}$} & \multirow{2}{*}{$\begin{array}{l}\text { Tem- } \\
\text { pera- } \\
\text { ture }\end{array}$} & \multicolumn{7}{|c|}{ Peak induction $k G$} \\
\hline & & & 2 & 4 & 6 & 8 & 10 & 12 & 14 \\
\hline $110 \mathrm{U}_{-.-.}$ & $\begin{array}{l}c / s \\
60\end{array}$ & $\begin{array}{r}{ }^{\circ} K \\
273 \\
195 \\
76 \\
4\end{array}$ & $\begin{array}{l}0.099 \\
1.34 \\
2.02 \\
2.42\end{array}$ & $\begin{array}{l}0.31 \\
1.39 \\
2.19 \\
2.65\end{array}$ & $\begin{array}{l}0.67 \\
1.36 \\
2.13 \\
2.60\end{array}$ & $\begin{array}{l}1.10 \\
1.35 \\
2.18 \\
2.73\end{array}$ & $\begin{array}{l}1.71 \\
1.35 \\
2.16 \\
2.63\end{array}$ & $\begin{array}{l}2.4 \\
1.29 \\
2.13 \\
2.67\end{array}$ & $\begin{array}{l}3.3 \\
1.27 \\
2.06 \\
2.55\end{array}$ \\
\hline $111 \mathrm{U}_{-. .}$ & 60 & $\begin{array}{r}273 \\
195 \\
76 \\
4\end{array}$ & $\begin{array}{l}0.089 \\
1.27 \\
2.10 \\
2.25\end{array}$ & $\begin{array}{l}0.28 \\
1.25 \\
2.21 \\
2.61\end{array}$ & $\begin{array}{l}0.54 \\
1.30 \\
2.31 \\
2.78\end{array}$ & $\begin{array}{l}0.89 \\
1.27 \\
2.25 \\
2.89\end{array}$ & $\begin{array}{l}1.40 \\
1.24 \\
2.21 \\
2.79\end{array}$ & $\begin{array}{l}\text { 1. } 9_{5} \\
\text { 1. } 23 \\
2.21 \\
2.82\end{array}$ & \\
\hline $111 B_{\ldots} \ldots$ & 60 & $\begin{array}{r}273 \\
195 \\
76 \\
4\end{array}$ & $\begin{array}{l}0.078 \\
1.08 \\
1.46 \\
2.18\end{array}$ & $\begin{array}{l}0.25 \\
1.12 \\
1.64 \\
2.32\end{array}$ & $\begin{array}{l}0.50 \\
1.10 \\
1.70 \\
2.48\end{array}$ & $\begin{array}{l}0.80 \\
1.15 \\
1.80 \\
2.63\end{array}$ & $\begin{array}{l}1.18 \\
1.16 \\
1.78 \\
2.71\end{array}$ & $\begin{array}{l}1.68 \\
1.15 \\
1.79 \\
2.80\end{array}$ & \\
\hline $112 \mathrm{U}_{-\ldots}$ & 60 & $\begin{array}{r}273 \\
195 \\
76 \\
4\end{array}$ & $\begin{array}{l}0.083 \\
1.22 \\
1.47 \\
1.69\end{array}$ & $\begin{array}{l}0.27 \\
1.26 \\
1.48 \\
1.67\end{array}$ & $\begin{array}{l}0.50 \\
1.28 \\
1.56 \\
1.78\end{array}$ & $\begin{array}{l}0.84 \\
1.21 \\
1.51 \\
1.81\end{array}$ & $\begin{array}{l}\text { 1. } 3_{1} \\
1.23 \\
1.51 \\
1.76\end{array}$ & $\begin{array}{l}1.83 \\
1.20 \\
1.48 \\
1.80\end{array}$ & \\
\hline $113 \mathrm{U}_{---}$ & 60 & $\begin{array}{r}273 \\
195 \\
76 \\
4\end{array}$ & $\begin{array}{l}0.083 \\
1.17 \\
1.36 \\
1.41\end{array}$ & $\begin{array}{l}0.25 \\
1.20 \\
1.40 \\
1.44\end{array}$ & $\begin{array}{l}0.50 \\
1.16 \\
1.36 \\
1.38\end{array}$ & $\begin{array}{l}0.82 \\
1.16 \\
1.33 \\
1.39\end{array}$ & $\begin{array}{l}1.27 \\
1.14 \\
1.31 \\
1.39\end{array}$ & $\begin{array}{l}1.80 \\
1.17 \\
1.28 \\
1.39\end{array}$ & \\
\hline $114 \mathrm{U}_{-}$ & 60 & $\begin{array}{r}273 \\
195 \\
76 \\
4\end{array}$ & $\begin{array}{l}0.064 \\
1.16 \\
1.30 \\
1.36\end{array}$ & $\begin{array}{l}0.21 \\
1.19 \\
1.33 \\
1.38\end{array}$ & $\begin{array}{l}0.39 \\
1.26 \\
1.38 \\
1.44\end{array}$ & $\begin{array}{l}0.66 \\
1.18 \\
1.32 \\
1.36\end{array}$ & $\begin{array}{l}1.03 \\
1.15 \\
1.27 \\
1.29\end{array}$ & $\begin{array}{l}\text { 1. } 50 \\
1.13 \\
1.23 \\
1.26\end{array}$ & \\
\hline $114 \mathrm{~B}_{\ldots}$ & 60 & $\begin{array}{r}273 \\
195 \\
76 \\
4\end{array}$ & $\begin{array}{l}0.056 \\
1.04 \\
1.11 \\
1.11\end{array}$ & $\begin{array}{l}0.195 \\
1.03 \\
1.08 \\
1.08\end{array}$ & $\begin{array}{l}0.39 \\
1.03 \\
1.08 \\
1.10\end{array}$ & $\begin{array}{l}0.64 \\
1.03 \\
1.08 \\
1.09\end{array}$ & $\begin{array}{l}0.96 \\
1.03 \\
1.08 \\
1.08\end{array}$ & & \\
\hline $114 \mathrm{~B}_{-}$ & 400 & $\begin{array}{r}273 \\
195 \\
76 \\
4\end{array}$ & $\begin{array}{l}0.69 \\
1.04 \\
1.14 \\
1.16\end{array}$ & $\begin{array}{l}2.5 \\
1.04 \\
1.12 \\
1.16\end{array}$ & $\begin{array}{l}5.3 \\
1.04 \\
1.13 \\
1.15\end{array}$ & $\begin{array}{l}9.2 \\
1.02 \\
1.12 \\
1.13\end{array}$ & $\begin{array}{l}\text { 14. } 0 \\
1.03 \\
1.16 \\
1.16\end{array}$ & $\begin{array}{l}20.0 \\
1.05 \\
1.15 \\
1.19\end{array}$ & \\
\hline $115 \mathrm{U}_{-. .}$ & 60 & $\begin{array}{r}273 \\
195 \\
76 \\
4\end{array}$ & $\begin{array}{l}0.072 \\
1.15 \\
1.40 \\
1.53\end{array}$ & $\begin{array}{l}0.24 \\
1.17 \\
1.42 \\
1.50\end{array}$ & $\begin{array}{l}0.47 \\
1.17 \\
1.43 \\
1.49\end{array}$ & $\begin{array}{l}0.77 \\
1.14 \\
1.38 \\
1.51\end{array}$ & $\begin{array}{l}\text { 1. } 17 \\
\text { 1. } 14 \\
\text { 1. } 38 \\
1.49\end{array}$ & $\begin{array}{l}\text { 1. } 69 \\
\text { 1.13 } \\
1.36 \\
1.42\end{array}$ & \\
\hline $116 \mathrm{U}_{-\ldots}$ & 60 & $\begin{array}{r}273 \\
195 \\
76 \\
4\end{array}$ & $\begin{array}{l}0.053 \\
1.26 \\
1.43 \\
1.45\end{array}$ & $\begin{array}{l}0.17_{5} \\
1.26 \\
1.37 \\
1.43\end{array}$ & $\begin{array}{l}0.34 \\
1.29 \\
1.41 \\
1.41\end{array}$ & $\begin{array}{l}0.55 \\
1.27 \\
1.36 \\
1.36\end{array}$ & $\begin{array}{l}0.83 \\
1.23 \\
1.27 \\
1.27\end{array}$ & $\begin{array}{l}\text { 1. } 18 \\
\text { 1. } 19 \\
1.30 \\
1.30\end{array}$ & \\
\hline $117 \mathrm{U}_{-}-.$. & 60 & $\begin{array}{r}273 \\
195 \\
76\end{array}$ & $\begin{array}{l}0.048 \\
1.23 \\
1.31\end{array}$ & $\begin{array}{l}0.15_{2} \\
1.21 \\
1.32\end{array}$ & $\begin{array}{l}0.30 \\
1.23 \\
1.33\end{array}$ & $\begin{array}{l}0.48 \\
1.21 \\
1.33\end{array}$ & $\begin{array}{l}0.74 \\
1.19 \\
1.31\end{array}$ & $\begin{array}{l}\text { 1. } 0_{3} \\
\text { 1.17 } \\
\text { 1. } 30\end{array}$ & \\
\hline $117 \mathrm{~B}_{-\ldots}$ & 60 & $\begin{array}{r}273 \\
195 \\
76 \\
4\end{array}$ & $\begin{array}{l}0.036 \\
1.06 \\
1.14 \\
1.17\end{array}$ & $\begin{array}{l}0.120 \\
1.03 \\
1.10 \\
1.13\end{array}$ & $\begin{array}{l}0.23 \\
1.04 \\
1.13 \\
1.13\end{array}$ & $\begin{array}{l}0.38 \\
1.03 \\
1.08 \\
1.08\end{array}$ & $\begin{array}{l}0.56 \\
1.04 \\
1.09 \\
1.11\end{array}$ & $\begin{array}{l}0.80 \\
1.04 \\
1.08 \\
1.09\end{array}$ & \\
\hline 117 B & 400 & $\begin{array}{r}273 \\
195 \\
76 \\
4\end{array}$ & $\begin{array}{l}0.42 \\
1.05 \\
1.12 \\
1.14\end{array}$ & $\begin{array}{l}1.43 \\
1.05 \\
1.10 \\
1.13\end{array}$ & $\begin{array}{l}2.8 \\
1.07 \\
1.14 \\
1.14\end{array}$ & $\begin{array}{l}\text { 4. } 7 \\
1.04 \\
1.11 \\
1.13\end{array}$ & $\begin{array}{l}7.3 \\
1.04 \\
1.10 \\
1.12\end{array}$ & $\begin{array}{c}10.6 \\
1.04 \\
1.10 \\
1.11\end{array}$ & \\
\hline $\begin{array}{l}118 \text { B (As } \\
\text { proc- } \\
\text { essed; } \\
\text { not an- } \\
\text { nealed). }\end{array}$ & 60 & $\begin{array}{r}273 \\
195 \\
76 \\
4\end{array}$ & $\begin{array}{l}0.039 \\
.97 \\
.95 \\
.95\end{array}$ & $\begin{array}{c}0.15_{2} \\
.99 \\
.97 \\
.96\end{array}$ & $\begin{array}{r}0.31 \\
.97 \\
.97 \\
.97\end{array}$ & $\begin{array}{r}0.51 \\
.98 \\
.96 \\
.96\end{array}$ & $\begin{array}{r}0.76 \\
.99 \\
.97 \\
.96\end{array}$ & $\begin{array}{l}\text { 1. } 01 \\
\text { 1. } 01 \\
\text { 1. } 02 \\
\text { 1. } 03\end{array}$ & \\
\hline $\begin{array}{l}118 \text { B (As } \\
\text { proc- } \\
\text { essed; } \\
\text { not an- } \\
\text { nealed). }\end{array}$ & 400 & $\begin{array}{r}273 \\
195 \\
76 \\
4\end{array}$ & $\begin{array}{l}0.48 \\
1.00 \\
1.00 \\
1.00\end{array}$ & $\begin{array}{l}1.80 \\
1.00 \\
1.00 \\
1.00\end{array}$ & $\begin{array}{l}3.9 \\
1.00 \\
1.00 \\
1.00\end{array}$ & $\begin{array}{l}6.0 \\
1.00 \\
1.00 \\
1.00\end{array}$ & $\begin{array}{l}\text { 11. } 0 \\
1.00 \\
1.00 \\
1.00\end{array}$ & $\begin{array}{l}\text { 16. } 3 \\
1.01 \\
1.02 \\
1.03\end{array}$ & $\begin{array}{c}25.0 \\
1.04 \\
1.04 \\
1.08\end{array}$ \\
\hline $\begin{array}{l}118 \text { B (An- } \\
\text { nealed). }\end{array}$ & 60 & $\begin{array}{r}273 \\
195 \\
76 \\
4\end{array}$ & $\begin{array}{l}0.035 \\
1.00 \\
1.00 \\
1.00\end{array}$ & $\begin{array}{l}0.11_{6} \\
1.00 \\
1.00 \\
1.00\end{array}$ & $\begin{array}{l}0.229 \\
1.00 \\
1.00 \\
1.00\end{array}$ & $\begin{array}{l}0.35 \\
1.00 \\
1.00 \\
1.00\end{array}$ & $\begin{array}{l}\text { 0. } 52 \\
\text { 1. } 00 \\
\text { 1. } 00 \\
\text { 1. } 00\end{array}$ & $\begin{array}{l}0.75 \\
1.00 \\
1.00 \\
1.00\end{array}$ & \\
\hline $\begin{array}{l}118 \text { B (An- } \\
\text { nealed). }\end{array}$ & 400 & $\begin{array}{r}273 \\
195 \\
76\end{array}$ & $\begin{array}{l}0.49 \\
1.00 \\
1.00\end{array}$ & $\begin{array}{l}1.80 \\
1.00 \\
1.00\end{array}$ & $\begin{array}{l}3.8 \\
1.00 \\
1.00\end{array}$ & $\begin{array}{l}5.8 \\
1.00 \\
1.00\end{array}$ & $\begin{array}{c}10.1 \\
1.00 \\
1.00\end{array}$ & $\begin{array}{c}\text { 14. } 3 \\
1.03 \\
1.06\end{array}$ & $\begin{array}{c}20.7 \\
1.04 \\
1.09\end{array}$ \\
\hline $212 \ldots$ & 60 & $\begin{array}{r}273 \\
195 \\
76 \\
4\end{array}$ & $\begin{array}{l}0.0072 \\
1.39 \\
1.83 \\
1.86\end{array}$ & $\begin{array}{l}0.025 \\
1.36 \\
1.88 \\
1.92\end{array}$ & & $\begin{array}{l}0.084 \\
1.33 \\
1.80 \\
1.92\end{array}$ & $\begin{array}{l}0.135 \\
1.29 \\
1.71 \\
1.84\end{array}$ & $\begin{array}{l}0.215 \\
1.27 \\
1.63 \\
1.67\end{array}$ & \\
\hline $212 \ldots$ & 400 & $\begin{array}{r}273 \\
195 \\
76 \\
4\end{array}$ & $\begin{array}{l}0.11_{2} \\
1.29 \\
1.70 \\
1.85\end{array}$ & $\begin{array}{l}0.39 \\
1.28 \\
1.72 \\
1.87\end{array}$ & & $\begin{array}{l}1.35 \\
1.26 \\
1.67 \\
1.83\end{array}$ & $\begin{array}{l}2.07 \\
1.30 \\
1.69 \\
1.84\end{array}$ & $\begin{array}{l}3.1 \\
1.26 \\
1.61 \\
1.81\end{array}$ & $\begin{array}{l}4.6 \\
1.24 \\
1.59 \\
1.74\end{array}$ \\
\hline
\end{tabular}

\begin{tabular}{|c|c|c|c|c|c|c|c|c|c|}
\hline \multirow{2}{*}{ Material } & \multirow{2}{*}{$\begin{array}{l}\text { Fre- } \\
\text { quen- } \\
\text { cy }\end{array}$} & \multirow{2}{*}{$\begin{array}{l}\text { Tem- } \\
\text { pera- } \\
\text { ture }\end{array}$} & \multicolumn{7}{|c|}{ Peak induction $k G$} \\
\hline & & & 2 & 4 & 6 & 8 & 10 & 12 & 14 \\
\hline ـ & $\begin{array}{c}c s \\
60\end{array}$ & $\begin{array}{r}{ }^{\circ} K \\
273 \\
195 \\
76 \\
4\end{array}$ & $\begin{array}{l}0.010_{8} \\
1.28 \\
1.69 \\
1.93\end{array}$ & $\begin{array}{l}0.038 \\
1.21 \\
1.74 \\
1.95\end{array}$ & & $\begin{array}{l}0.138 \\
1.22 \\
1.70 \\
1.96\end{array}$ & $\begin{array}{l}0.224 \\
1.21 \\
1.70 \\
1.88\end{array}$ & $\begin{array}{l}0.33 \\
1.30 \\
1.76 \\
1.91\end{array}$ & \\
\hline 213 & 400 & $\begin{array}{r}273 \\
195 \\
76 \\
4\end{array}$ & $\begin{array}{l}\text { 0. } 21_{1} \\
\text { 1. } 23 \\
\text { 1. } 75 \\
\text { 1. } 94\end{array}$ & $\begin{array}{l}0.78 \\
1.21 \\
1.69 \\
1.90\end{array}$ & & $\begin{array}{l}3.5 \\
1.20 \\
1.71 \\
1.91\end{array}$ & $\begin{array}{l}6.2 \\
1.24 \\
1.66 \\
1.85\end{array}$ & $\begin{array}{l}9.2 \\
1.29 \\
1.76 \\
1.99\end{array}$ & $\begin{array}{c}12.3 \\
1.38 \\
1.91 \\
2.11\end{array}$ \\
\hline
\end{tabular}

With the exception of sample 118 all materials were stress-relief annealed by the fabricators following their normal annealing procedures. Sample 118 was tested in both unannealed, as fabricated, and annealed conditions. Both unannealed and annealed samples were boxed to prevent the extraneous effects of winding stresses. The temperature behavior of these two samples will be discussed later in relation to theories of coercive force.

Internal heating was, in some instances, great enough to raise the sample temperature above that of the environmental bath. The temperature rise was determined using a $\mathrm{Au}-\mathrm{Co}$ versus copper thermocouple. Core loss values presented in table 3 have been interpolated where necessary to give loss values at the bath temperatures listed.

\section{Alternating Current Loss Data}

Alternating current core loss for each sample was measured at $4,76,195$, and $273{ }^{\circ} \mathrm{K}$ for various peak inductions from 2 to $18 \mathrm{~kg}^{2}$ All materials were tested at $60 \mathrm{c} / \mathrm{s}$ (hertz). Where the type of material warranted it, measurements were also made at 400 and $1000 \mathrm{c} / \mathrm{s}$. At $60 \mathrm{c} / \mathrm{s}$ the upper induction level is limited by waveform distortion. At $1000 \mathrm{c} / \mathrm{s}$ it is limited by the breakdown voltage of the magnet wire insulation. In the interest of saving space, only four graphs (figs. 1-4) showing core loss versus temperature are presented. The bulk of the data are presented in table 3.

From the data given in figures 1 to 4 and table 3 it is noted that the high silicon content (3 to $4.45 \%$ $\mathrm{Si}$ ) Fe-Si alloys are the least sensitive to temperature variations. This is primarily a result of these alloys having a lesser temperature dependence of electrical resistivity resulting in a smaller increase in eddy current loss. Although there has been some criticism in the past of separating total core loss into hysteresis loss and eddy current loss components, we find that it is helpful, at least qualitatively, to speak in terms of a separate hysteresis and eddy current loss.

${ }_{2}^{2}$ The following relationships may be used to obtain equivalent values in the MKSA system of units.

$$
\begin{array}{ll}
1 \text { kilogauss } & =0.1 \text { tesla } \\
1 \text { oersted } & =79.58 \text { ampere turns } / \text { meter } \\
1 \mathrm{watt} / \text { pound } & =2.204 \mathrm{watt} / \mathrm{kilogram} \\
1 \mathrm{inch} & =0.02540 \text { meter }
\end{array}
$$




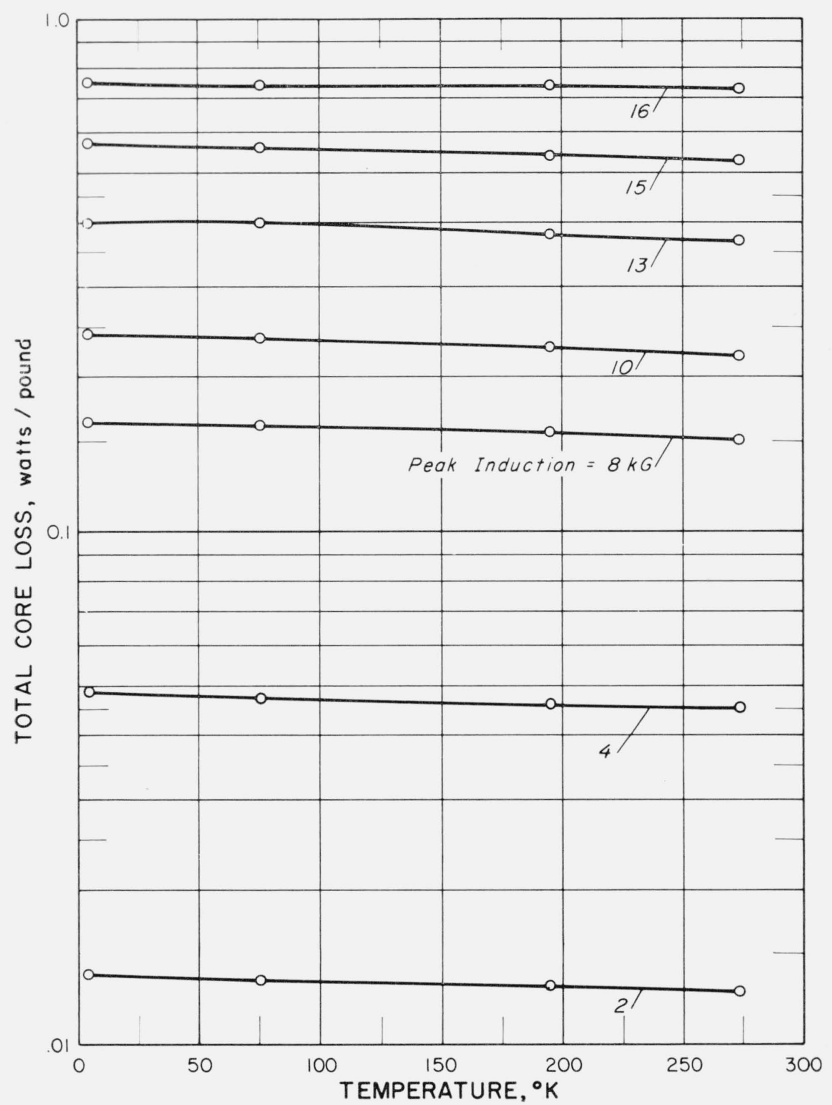

Figure 1. Total core loss at $60 \mathrm{c} / \mathrm{s}$ for various peak inductions: Material 102.

For instance, upon examining the data for material 102 in table 3 , it is noted that at $60 \mathrm{c} / \mathrm{s}$ and for low peak inductions there is a loss increase of approximately 8 percent upon cooling from 273 to $4{ }^{\circ} \mathrm{K}$. At $400 \mathrm{c} / \mathrm{s}$ and $1000 \mathrm{c} / \mathrm{s}$ however, the corresponding loss increase is approximately 20 percent. This difference may be explained by assuming the greater part of the total loss at $60 \mathrm{c} / \mathrm{s}$ to be due to a hysteresis loss while at 400 and $1000 \mathrm{c} / \mathrm{s}$ an increasing percentage of the total loss is due to eddy current losses which, to a first approximation, may be expected to vary inversely as the material resistivity. From the resistance data given in figure 5 it is seen that for material $102, R_{4} / R_{273}=0.82$. This gives an expected eddy current loss increase of approximately 22 percent which is in good agreement with the total core loss data at $1000 \mathrm{c} / \mathrm{s}$. By similar arguments involving separate hysteresis and eddy current loss components a satisfactory comparison of the temperature dependence of core loss can be made among materials 100,101 , and 102 which are similar except for tape thickness.

As a result of this study it may be said that, in general, a-c core loss will increase upon cooling from 273 to $4{ }^{\circ} \mathrm{K}$. The amount of increase varies

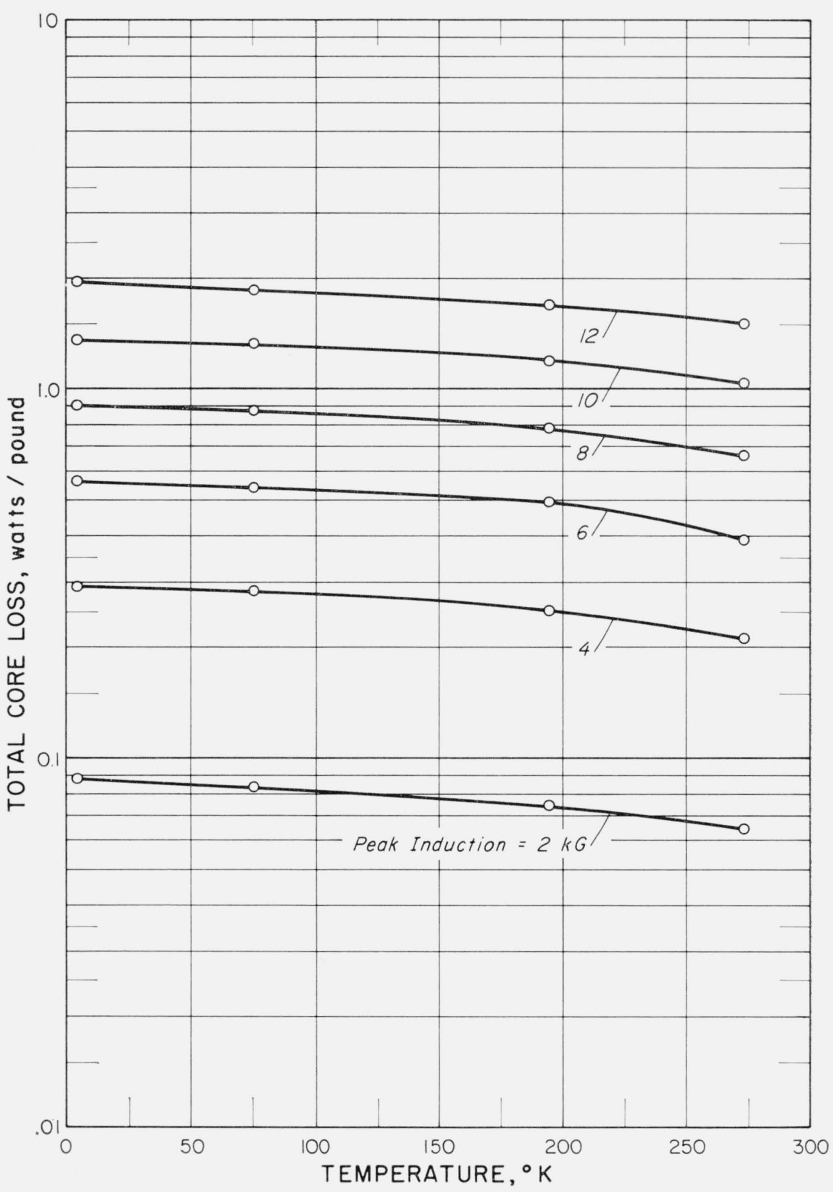

Figure 2. Total core loss at $60 \mathrm{c} / \mathrm{s}$ for various peak inductions: Material 114 unboxed.

greatly from one material to another, the range being from nearly no increase to almost 200 percent for the materials tested. The one exception to the above statement is material 118. As seen in table 3 this sample, in the unannealed condition, showed a loss decrease of approximately 4 percent at $60 \mathrm{c} / \mathrm{s}$. This decrease with decreasing temperatures is also evident in the d-c hysteresis loss measurements and will be discussed further in the section on d-c properties.

\section{Direct Current Properties}

Direct current hysteresis loops at peak inductions of 5,10 , and $15 \mathrm{kG}$ were measured for the same samples used for a-c measurements. Values of the coercive force, $H_{c}$, and the ratio of residual induction to peak induction, $B_{r} / B_{m}$, were measured directly from the loops and should be accurate to within approximately 1 percent. The d-c hysteresis loss, $P_{h}$, was determined from planimeter measurements of the loop area and should be accurate to about 1 to 2 percent except for induction levels which required a change of scale in the 


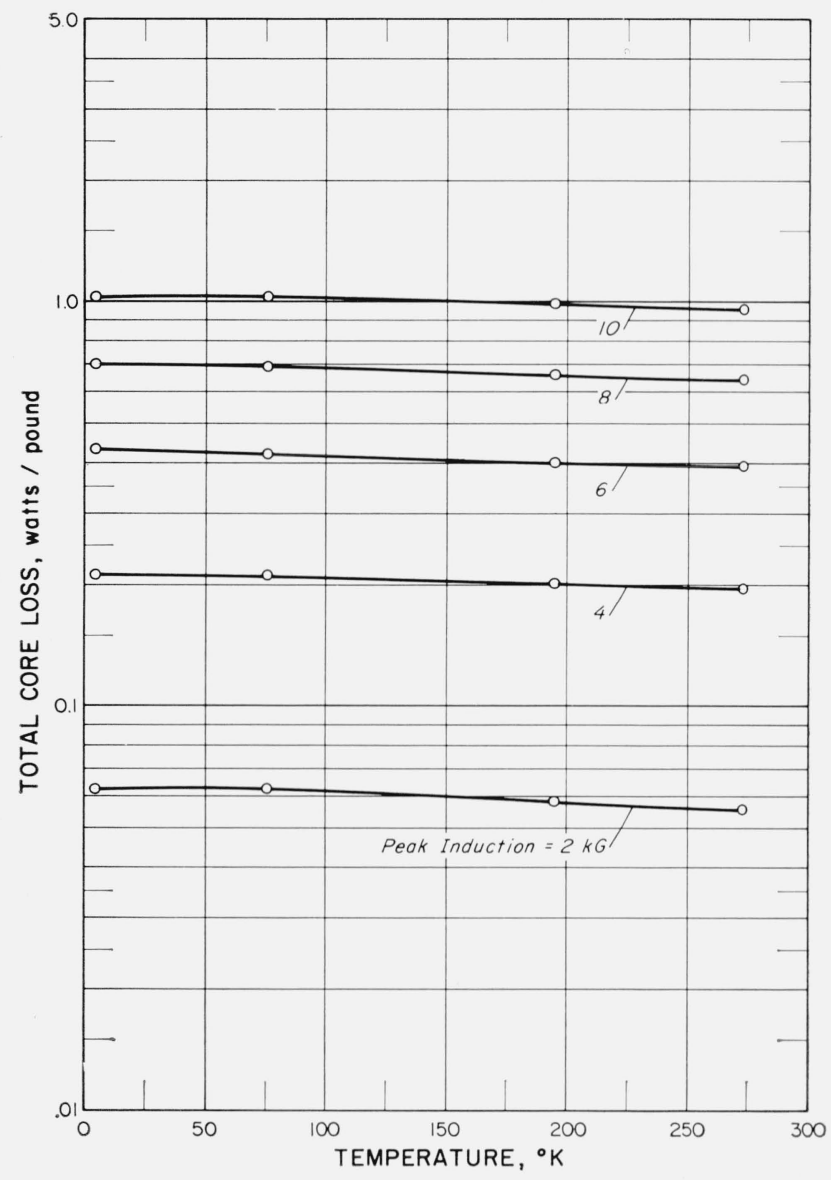

Figure 3. Total core loss at $60 \mathrm{c} / \mathrm{s}$ for various peak inductions: Material 114 boxed.

$H$-axis. In the latter cases the loss values may be in error up to approximately 5 percent.

Inasmuch as the winding stresses are known to influence the hysteresis loss temperature dependence, only the boxed samples will be considered here. The effects observed are therefore of purely magnetic origin. The results for the $\mathrm{Fe}-\mathrm{Si}$ and $\mathrm{Fe}-\mathrm{Ni}$ alloys are presented separately.

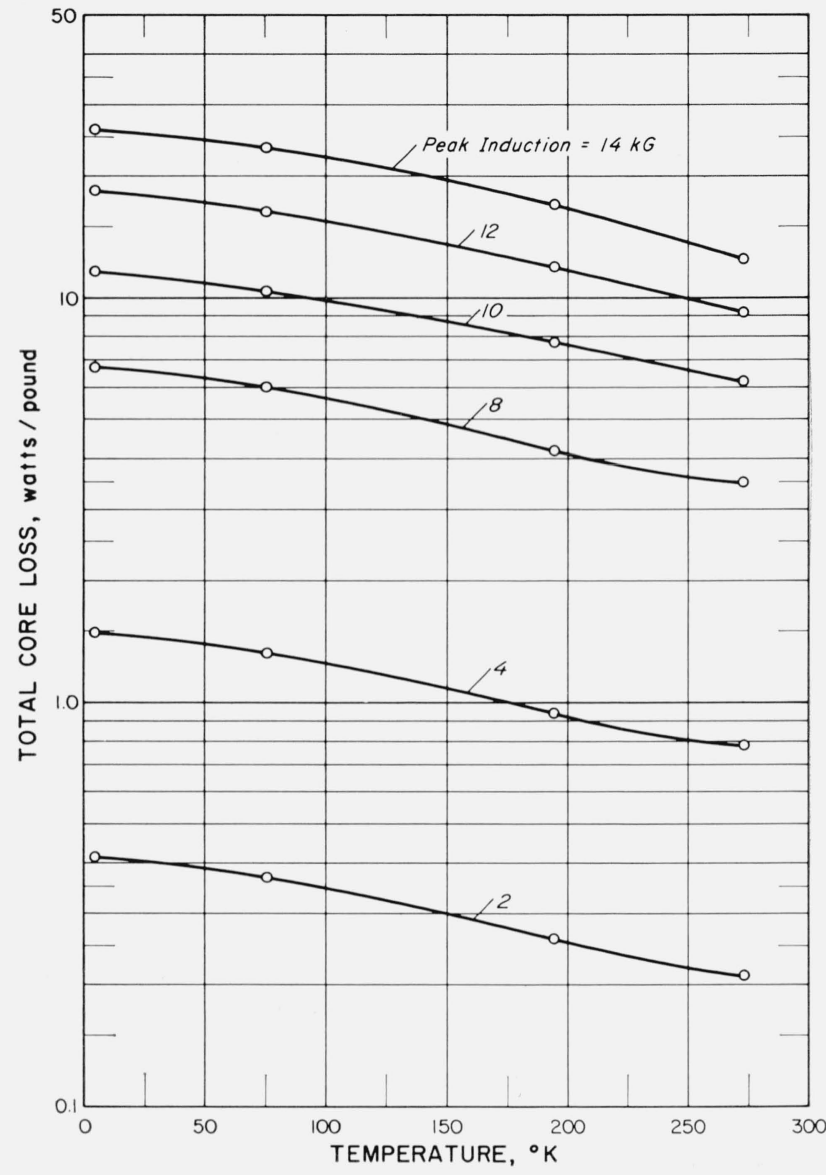

Figure 4. Total core loss at $400 \mathrm{c} / \mathrm{s}$ for various peak inductions: Material 213.

\subsection{Fe-Si Alloys}

The temperature dependence of the coercive force, residual induction, and hysteresis loss is shown graphically in figures 6 through 10 with the results normalized to 1.00 at $273^{\circ} \mathrm{K}$. For comparison purposes the absolute values of $H_{c}, B_{r} / B_{m}$, and $P_{h}$ at $273^{\circ} \mathrm{K}$ are tabulated in table 4 .

TABLE 4. Coercive force $\left(\mathrm{H}_{\mathrm{c}}\right)$, ratio of residual induction to peak induction $\left(\mathrm{B}_{\mathrm{r}} / \mathrm{B}_{\mathrm{m}}\right)$, and hysteresis loss $\left(\mathrm{P}_{\mathrm{h}}\right)$ at $273{ }^{\circ} K$

\begin{tabular}{|c|c|c|c|c|c|c|c|c|c|}
\hline \multirow{2}{*}{ Sample } & \multicolumn{3}{|c|}{$5 \mathrm{kG}$} & \multicolumn{3}{|c|}{$10 \mathrm{kG}$} & \multicolumn{3}{|c|}{$15 \mathrm{kG}$} \\
\hline & $H_{c}$ & $B_{r} / B_{m}$ & $P_{h}$ & $H_{0}$ & $B_{r} / B_{m}$ & $P_{h}$ & $H_{c}$ & $B_{r} / B_{m}$ & $P_{h}$ \\
\hline $\begin{array}{l}100 \\
101 \\
111 \\
112 \\
114 \\
117 \\
118 \text { unannealed annealed }\end{array}$ & $\begin{array}{r}O e \\
0.442 \\
.241 \\
.136 \\
.482 \\
.388 \\
.471 \\
.279 \\
.423 \\
.231\end{array}$ & $\begin{array}{l}0.652 \\
.660 \\
.634 \\
.820 \\
.811 \\
.743 \\
.783 \\
.624 \\
.512\end{array}$ & $\begin{array}{c}\text { erg/cm3/cycle } \\
537 \\
290 \\
163 \\
661 \\
541 \\
621 \\
386 \\
517 \\
281\end{array}$ & $\begin{array}{c}O e \\
0.670 \\
.345 \\
.187 \\
.610 \\
.485 \\
.630 \\
.368 \\
.563 \\
.303\end{array}$ & $\begin{array}{l}0.843 \\
.844 \\
.810 \\
.892 \\
.865 \\
.822 \\
.872 \\
.672 \\
.396\end{array}$ & $\begin{array}{c}\mathrm{erg} / \mathrm{cm}^{3} / \mathrm{cycle} \\
1956 \\
981 \\
507 \\
1937 \\
1603 \\
2053 \\
1163 \\
1664 \\
821\end{array}$ & $\begin{array}{c}O e \\
0.952 \\
.470 \\
.234 \\
.719 \\
.582 \\
.735 \\
.426 \\
.632 \\
.325\end{array}$ & $\begin{array}{r}0.880 \\
.909 \\
.958 \\
.885 \\
.838 \\
.744 \\
.802 \\
.564 \\
.291\end{array}$ & $\begin{array}{c}\mathrm{erg} / \mathrm{cm}^{3} / \mathrm{cycle} \\
5871 \\
2534 \\
1148 \\
5991 \\
4161 \\
6716 \\
3284 \\
4517\end{array}$ \\
\hline
\end{tabular}




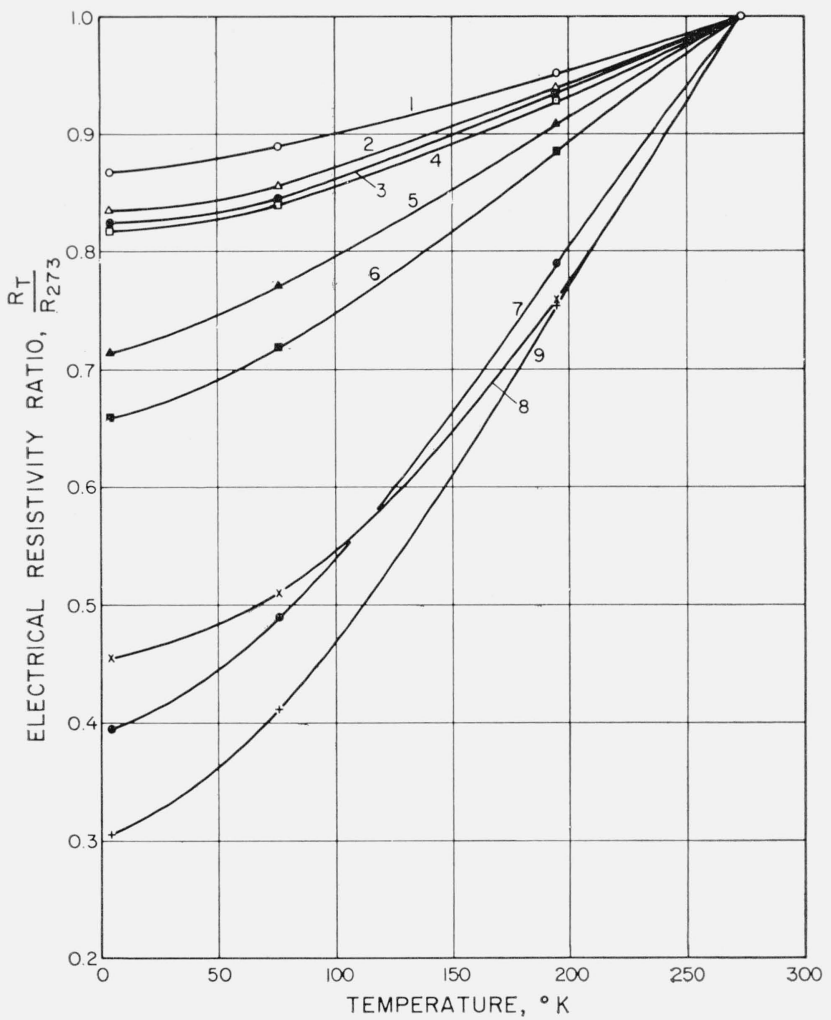

Figure 5. Electrical resistances, ratios of low temperature to ice-point values.

The numbered curves are for the following materials listed in tables 1 and 2 ; (1) $118 ;$ (2) 117 ; (3) 116 ; (4) 113 and $100,101,102$; (5) 114 ; (6) 112 ; (7) 110 ; (8) 200 series; (9) 111.

\section{a. Hysteresis Loss}

The magnetization process in ferromagnets is commonly described in terms of domain wall motion and domain rotation processes. By measuring the d-c hysteresis loops at peak inductions of 5, 10, and $15 \mathrm{kG}$ it was intended to proceed from an induction level $(5 \mathrm{kG})$ where comparatively simple domain wall motion processes predominate to an induction level $(15 \mathrm{kG})$ where domain rotations and the more complex closure domains [3] become increasingly important. It is of course realized that this somewhat arbitrary choice of peak induction levels will distinguish between the different magnetization processes to varying degrees for the various materials tested. To illustrate this it would be well to consider materials 100 and 102 . The hysteresis loops at a peak induction of $15 \mathrm{kG}$ are shown for these two materials in figures 11 and 12 , respectively. It is evident from the loops that the magnetization of material 102 has proceeded primarily by simple domain wall motion. On the other hand, the curve for material 100 suggests that the magnetization process is well into the region of domain rotations and the associated closure domain wall movements. This influence of magnetization process on the temperature dependence of the hysteresis loss is shown

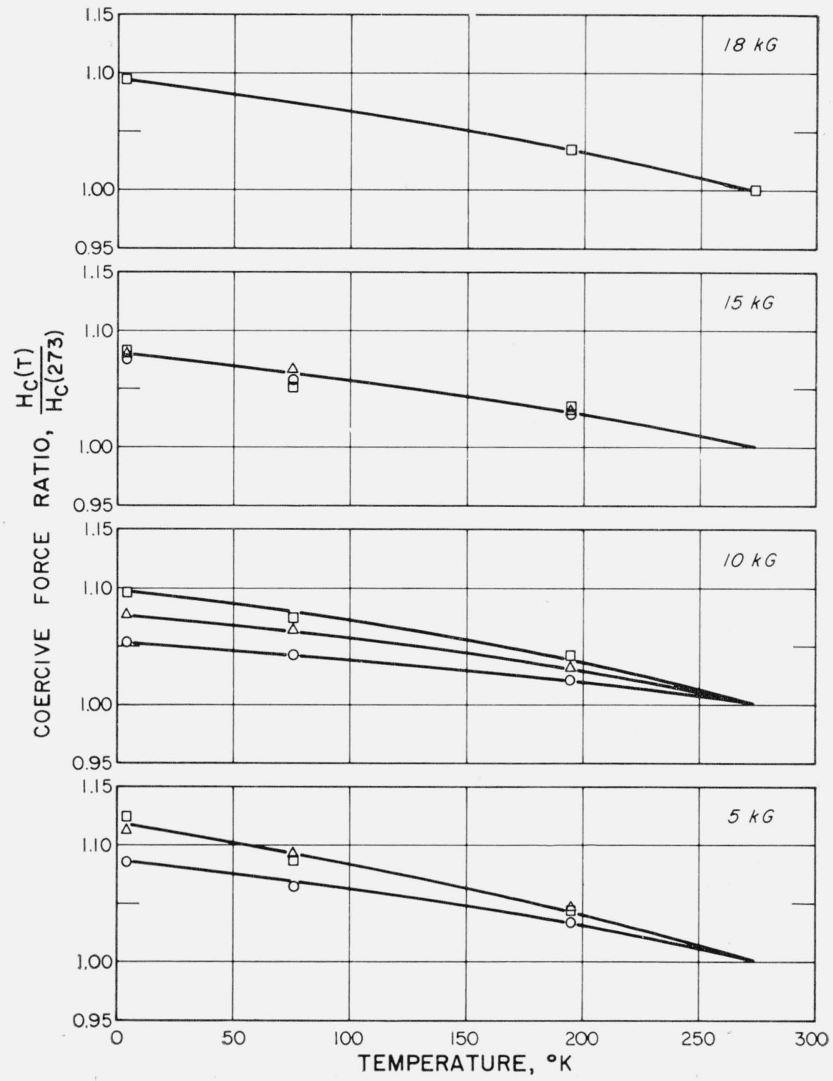

FiguRE 6. Coercive force, ratio of low temperature to ice-point values.

Grain-oriented material: $\bigcirc 100 ; \triangle 101 ; \square 102$. Materials identified in table 1.

in figure 7 . Here it is noted that at $15 \mathrm{kG}$, material 100 exhibits a temperature independent hysteresis loss. This corresponds to Chen's statement [3] that at the higher temperatures the decrease in energy loss associated with irreversible domain wall movement may be completely compensated by an increase in closure domain loss. At $15 \mathrm{kG}$ material 102 still shows an increased hysteresis loss at $4{ }^{\circ} \mathrm{K}$. The increase, however, is only about 5 percent compared to 10 percent at $10 \mathrm{kG}$ and approximately 13 percent at $5 \mathrm{kG}$. Thus it indicates that the magnetization of material 102 is just starting to enter the region of domain rotation at $15 \mathrm{kG}$. It would be expected that at some higher peak induction material 102 would exhibit a temperature behavior comparable to the behavior of material 100 at $15 \mathrm{kG}$. This in fact is the case at $18 \mathrm{kG}$ where the hysteresis loss for material 102 was measured to be approximately 2 percent lower at $4{ }^{\circ} \mathrm{K}$ compared to the $273{ }^{\circ} \mathrm{K}$ value. Ignoring material 118 for the moment it is found that a similar situation exists for the unoriented alloys as shown in figure 9. While there is a hysteresis loss increase of approximately 4 percent at $76{ }^{\circ} \mathrm{K}$ for both 5 and $10 \mathrm{kG}$ the hysteresis loss decreases by approximately 2 percent for a peak induction of $15 \mathrm{kG}$. 


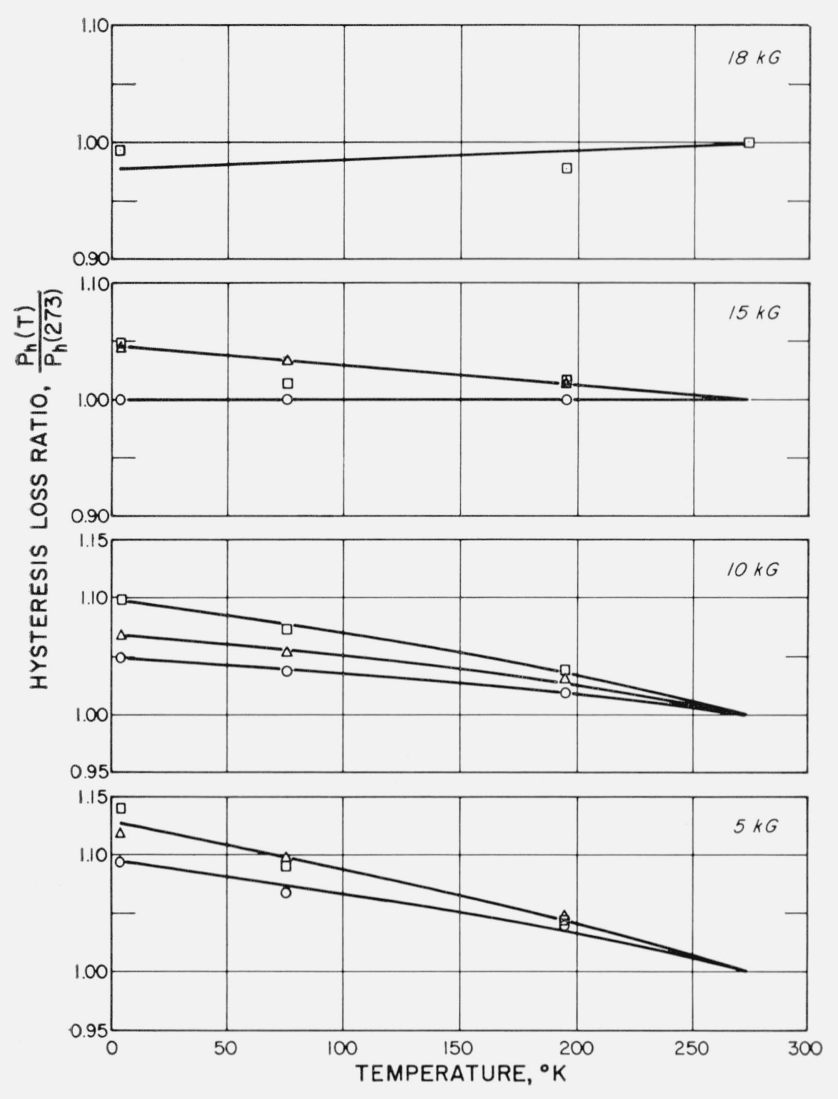

Figure 7. Hysteresis loss, ratio of low temperature to ice-point values.

Grain-oriented material: $\bigcirc 100 ; \triangle 101 ; \square$ 102. Materials identified in table 1.

\section{b. Coercive Force}

Upon comparison of the curves for the temperature dependence of coercive force and hysteresis loss it is found for materials 100-102 that for peak inductions of 5 and $10 \mathrm{kG}$ the temperature behavior is nearily identical. Thus the hysteresis loss behavior is determined almost entirely by the temperature dependence of the coercive force. At $15 \mathrm{kG}(18 \mathrm{kG}$ for material 102), there is no such correspondence. This is due to the effect of closure domain loss as discussed previously. It should be observed, however, that the increase in coercive force at $4^{\circ} \mathrm{K}$ is very nearly the same for both the 10 and $15 \mathrm{kG}$ levels of induction.

\section{c. Residual Induction}

As discussed by Chen the residual induction may be expected to increase with decreasing temperature. The primary reason for this is the temperature dependence of the magnetocrystalline anisotropy, $K$, in the expression for the wall energy. Inasmuch as this model is concerned with the nucleation of new reverse domains, the expression for wall energy would be expected to give an indication of the temperature dependence of the residual induction

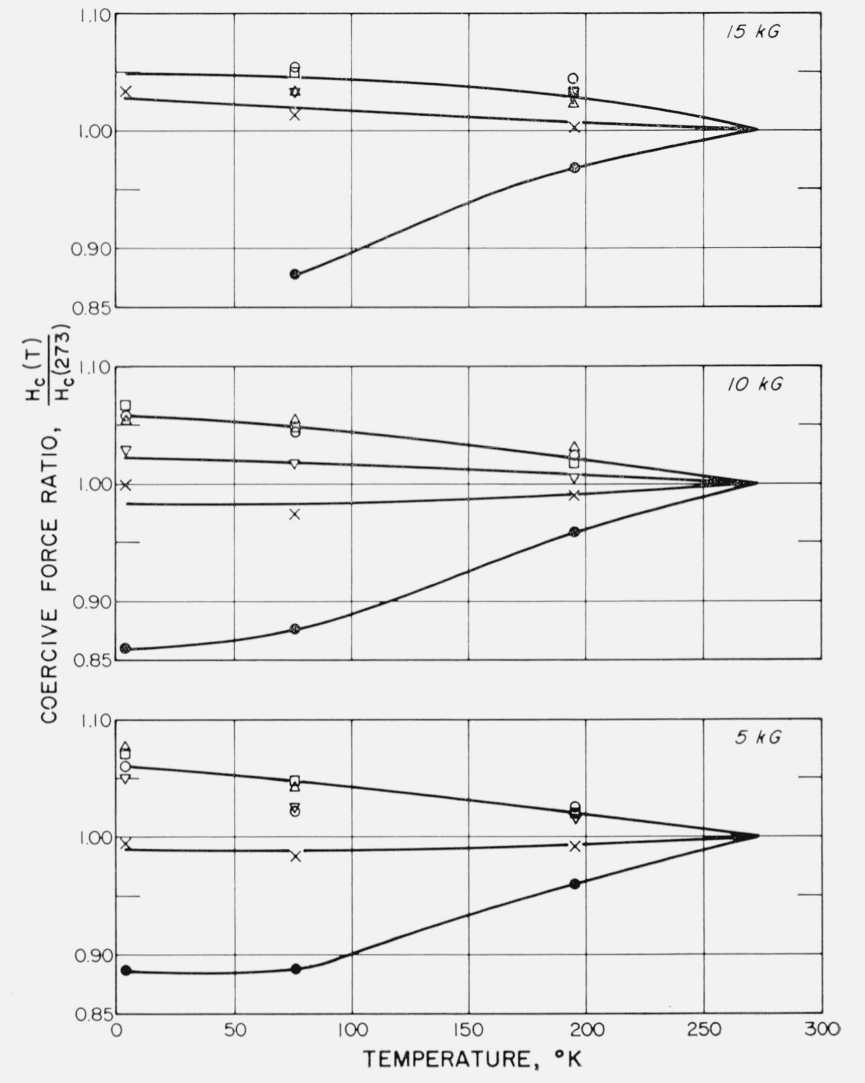

Figure 8. Coercive force, ratio of low temperature to ice-point values.

Boxed unoriented material: $\bigcirc 111 ; \triangle 112 ; \square 114 ; \nabla 117, \bullet 118$, unannealed: $\times 118$ annealed. Materials identified in table 1 .

only at induction levels approaching saturation where all the original domain walls have been eliminated. Indeed, for the samples shown in figure 10, with the exception of 118, the residual induction is essentially temperature independent at the lower peak inductions of 5 and $10 \mathrm{kG}$.

\section{d. Material 118}

As can be seen from the curves for $H_{c}, B_{r}$, and $P_{h}$, material 118 exhibits a behavior different from the other iron-silicon alloys tested. For this reason it is presented separately. We have no complete explanation for the temperature behavior of this material but feel it is probably associated with the vanishing magnetostriction constant at low temperatures for a silicon content of 4.45 percent [4].

As discussed by Chen the current theories indicate the coercive force to be a function of $\lambda_{s} / I_{s}$ or $K / I_{s}$ or both, where $\lambda_{s}$ is the magnetostriction constant, $I_{s}$ is the saturation magnetization, and $K$ is the magnetocrystalline anisotropy. Whereas Chen considered the energy term associated with magnetostriction to be negligible compared to the crystal anisotropy term, this is not the case for 4.45 percent silicon iron. In the case of the unannealed, "as processed" sample, the material is undoubtedly severely stressed. 

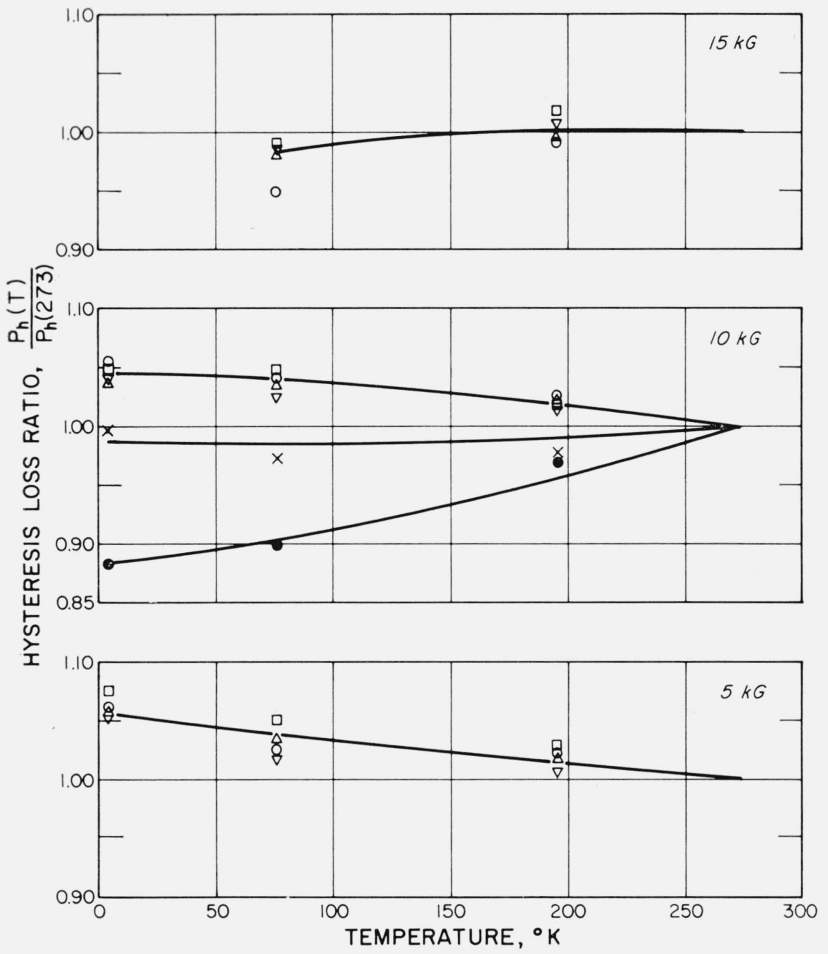

Figure 9. Hysteresis loss, ratio of low temperature to ice-point values.

Boxed unoriented material: $\bigcirc 111: \triangle 112: \square$ 114: $\nabla$ 117: • 118 unannealed; $X$ 118 annealed. Materials identified in table 1.

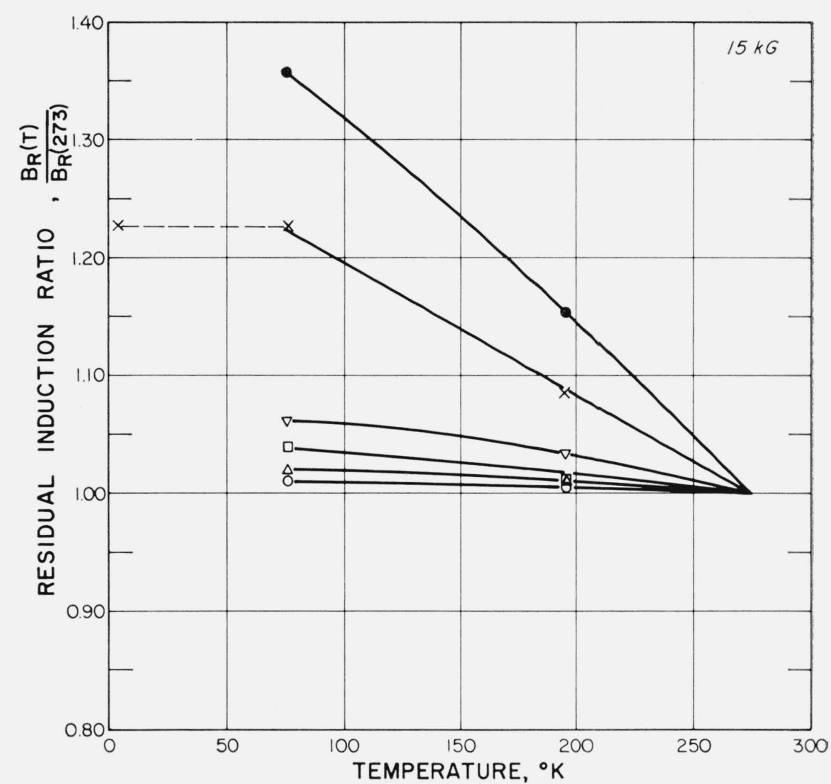

Figure 10. Residual induction, ratio of low temperature to ice-point value.

Boxed unoriented material: $\bigcirc 111 ; \triangle 112 ; ' \square$ 114; $\nabla$ 117; • 118 unannealed; $X$ 118 annealed. Materials identified in table 1.

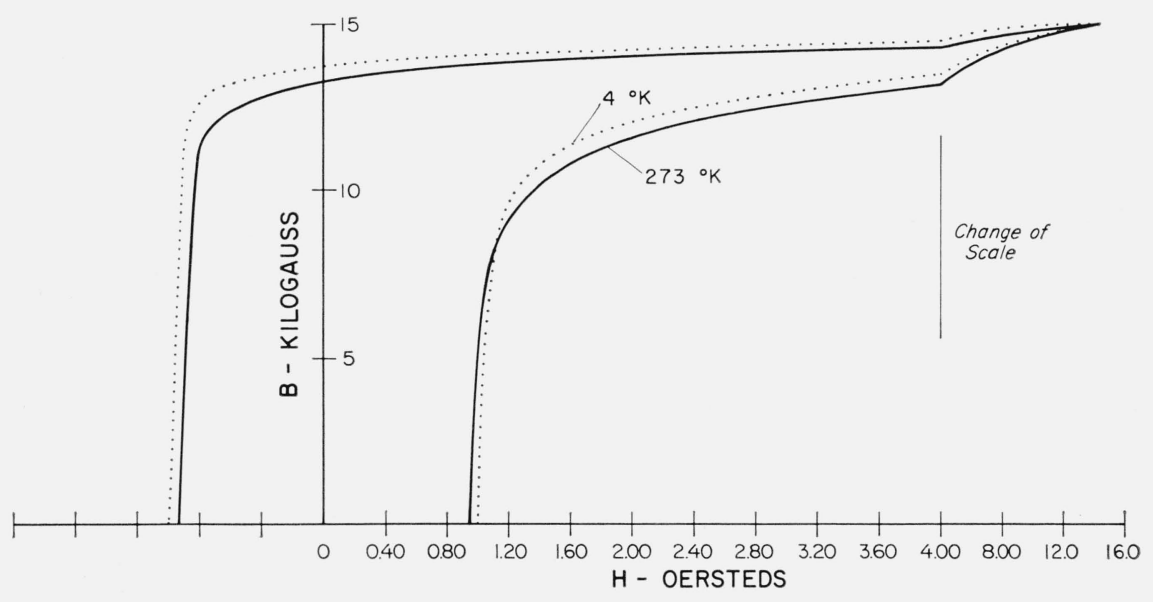

Figure 11. Hysteresis loop at a peak induction of $15 k G$. Material 100.

Taking the internal stress to be of the order of the yield stress $\left(4.9 \times 10^{9} \mathrm{dyn} / \mathrm{cm}^{2}\right)$ and $\lambda_{100}=2.0 \times 10^{-5}$, the energy associated with magnetostriction is $9.8 \times 10^{4} \mathrm{ergs} / \mathrm{cm}^{3}$ compared to a crystal anisotropy energy of $30 \times 10^{4} \mathrm{ergs} / \mathrm{cm}^{3}$ at room temperature. Clearly the magnetostriction energy is not negligible.
The decrease in $H_{c}$ shown in figure 8 is thus attributed to the decrease in the magnetostriction constant upon cooling below $273^{\circ} \mathrm{K}$.

If this interpretation is correct the removal of internal stresses by annealing would be expected to result in a temperature dependence of coercive force 


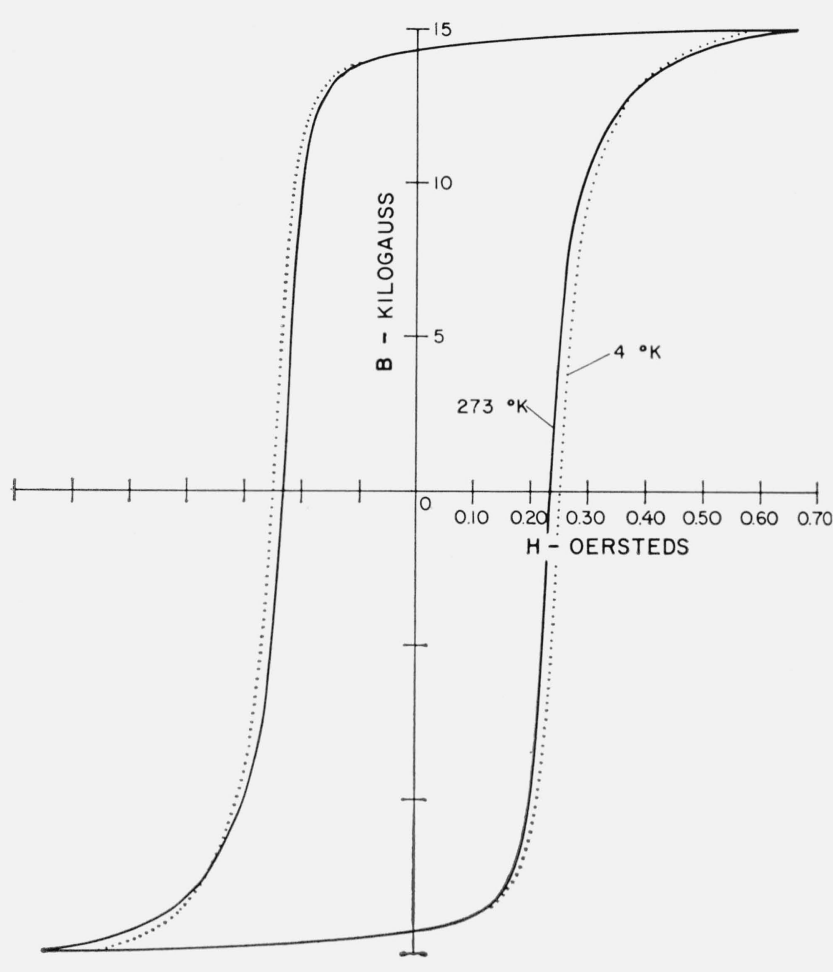

Figure 12. Hysteresis loop at a peak induction of $15 k G$. Material 102.

determined by the crystal anisotropy energy only. As shown for the annealed sample this result was partially realized; the coercive force at $15 \mathrm{kG}$ increased slightly with decreasing temperature. It should be noted that the coercive force at $5 \mathrm{kG}$ and $10 \mathrm{kG}$ still shows a small decrease at the lower temperatures. The decrease is, however, much less than for the unannealed sample. It is possible that annealing the material at $1425^{\circ} \mathrm{F}$ for $24 \mathrm{hr}$ does not completely remove the internal stresses, thus leaving a small dependence on the magnetostriction energy. Annealing at higher temperatures was not attempted since it was feared that recrystallization might occur thereby introducing a new variable of crystal orientation [5].

The behavior of the residual induction for both the unannealed and annealed material is shown in figure 10. The unannealed sample shows a greater temperature dependence than the annealed material. For a material whose magnetostriction decreases with decreasing temperature this would not be expected to occur on the basis of the wall energy equation mentioned previously. Barring the possibility of recrystallization occurring during the annealing treatment one must conclude that it is an oversimplification to treat the residual induction in terms of the wall nucleation process only.

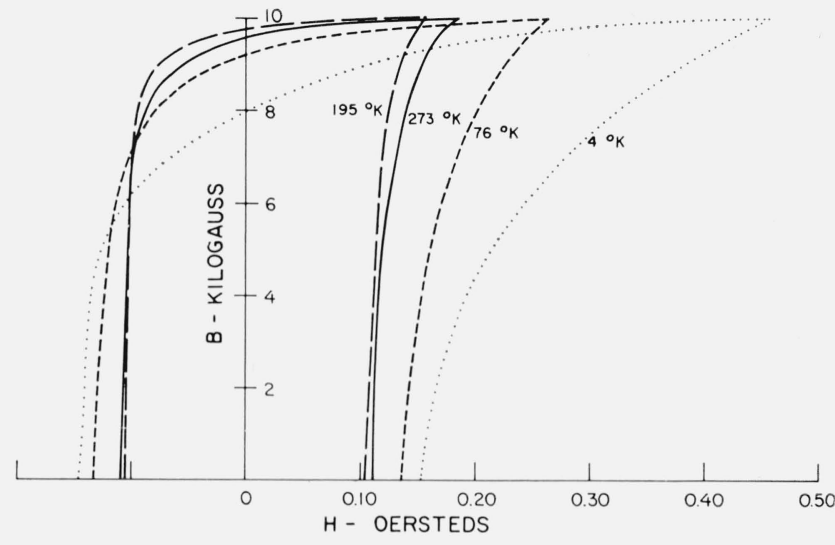

Figure 13. Hysteresis loops. Material 200.

\subsection{Fe-Ni Alloys}

The iron-nickel alloys tested are less well-behaved than the iron-silicon alloys discussed in the previous section. Their behavior is not completely understood at present. In view of their potential use at cryogenic temperatures however, and the large changes in the magnetic properties which were found to occur, some results are presented here without explanation. The curves are intended primarily for design and application use.

Hysteresis loops for a variety of materials are presented in figures 13 through 20. The curves shown in figure 17 are for a material commercially supplied as $0.002 \mathrm{in.} 48 \mathrm{Ni}$. However, metallurgical investigation indicated a very small grain size which is not considered to be typical for this material. The curves are presented in view of the strange behavior evident at $76^{\circ} \mathrm{K}$. It is not known whether such a temperature behavior would be the rule or the exception for commercially supplied material of this type. A laboratory prepared sample, of 0.002 in. $48 \mathrm{Ni}$, supplied by Armeo Steel, was also tested. The results are shown in figure 18 where it is seen that the kinked loop behavior is absent.

\section{Summary}

The a-c and d-c magnetic properties of selected $\mathrm{Fe}-\mathrm{Si}$ and $\mathrm{Fe}-\mathrm{Ni}$ alloys have been measured at cryogenic temperatures. The results are presented in tables and graphs to provide engineering design data. Of the Fe-Si alloys, the thin grain-oriented material would be best suited for cryogenic use. In addition to its superior room temperature properties, it exhibits a considerably lower loss increase at cryogenic temperatures than do the thicker laminated, low silicon content alloys. The $\mathrm{Fe}-\mathrm{Ni}$ alloys are in 


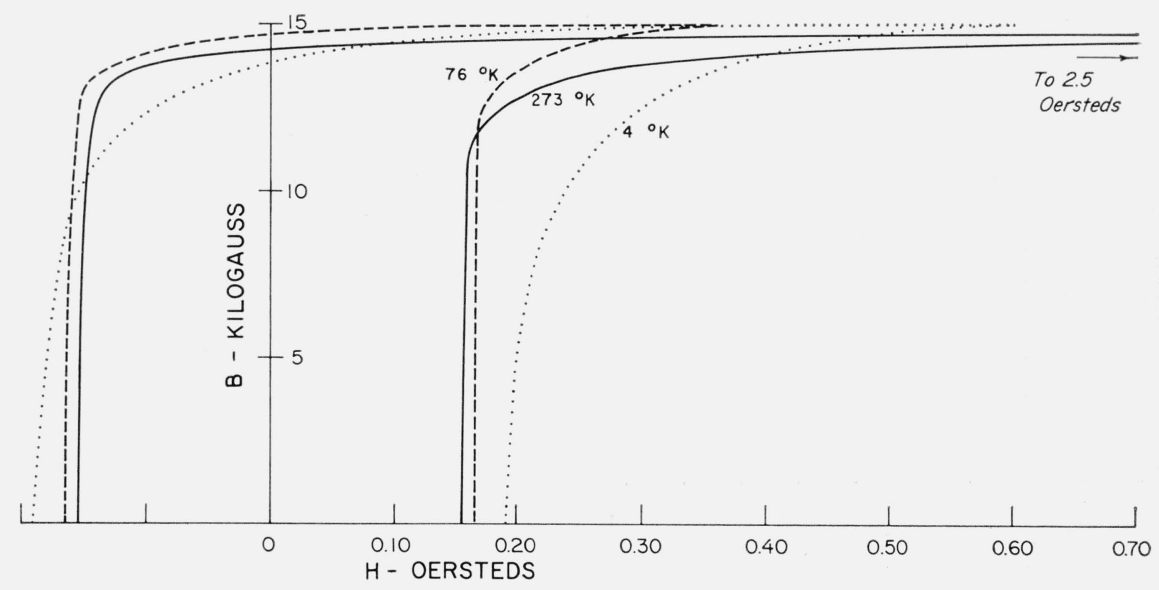

Figure 14. Hysteresis loops.

Material 201.

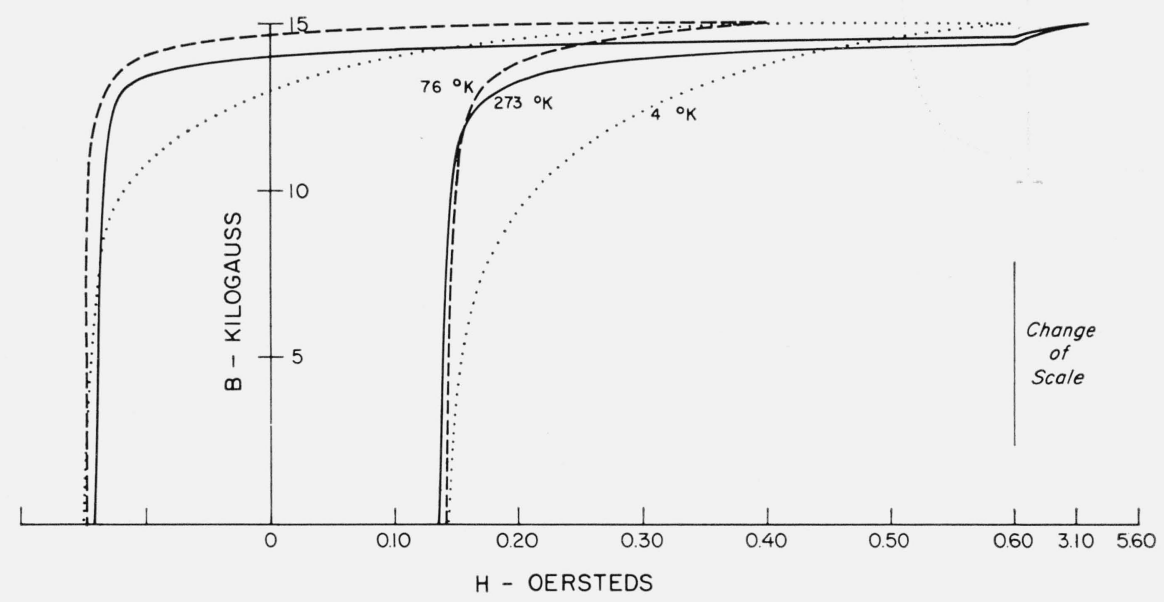

Figure 15. Hysteresis loops.

Material 202.

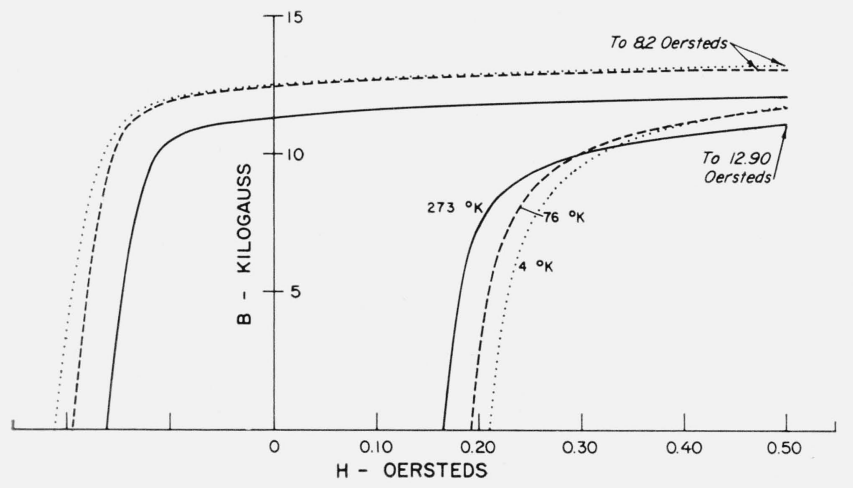

Figure 16. Hysteresis loops. Material 203.

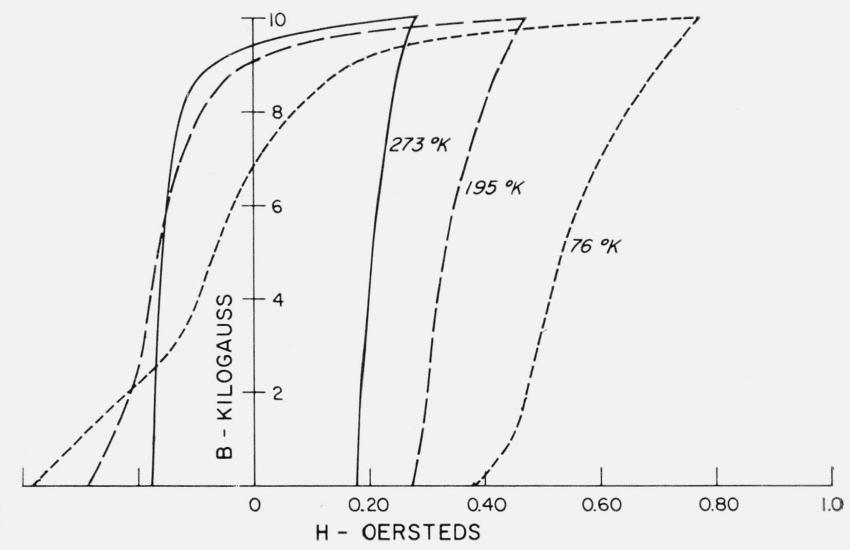

Figure 17. Hysteresis loops.

Material 210; commercial preparation. 


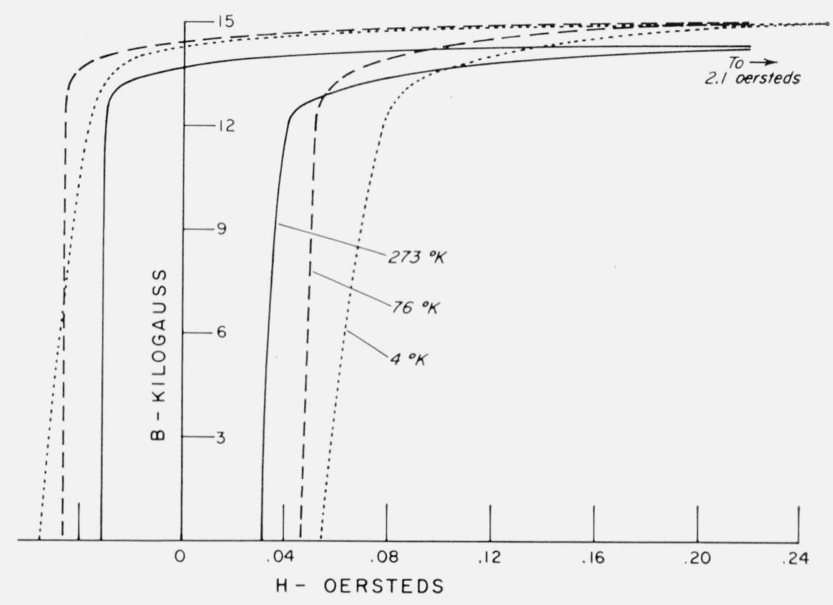

Figure 18. Hysteresis loops.

Material 210; laboratory preparation.

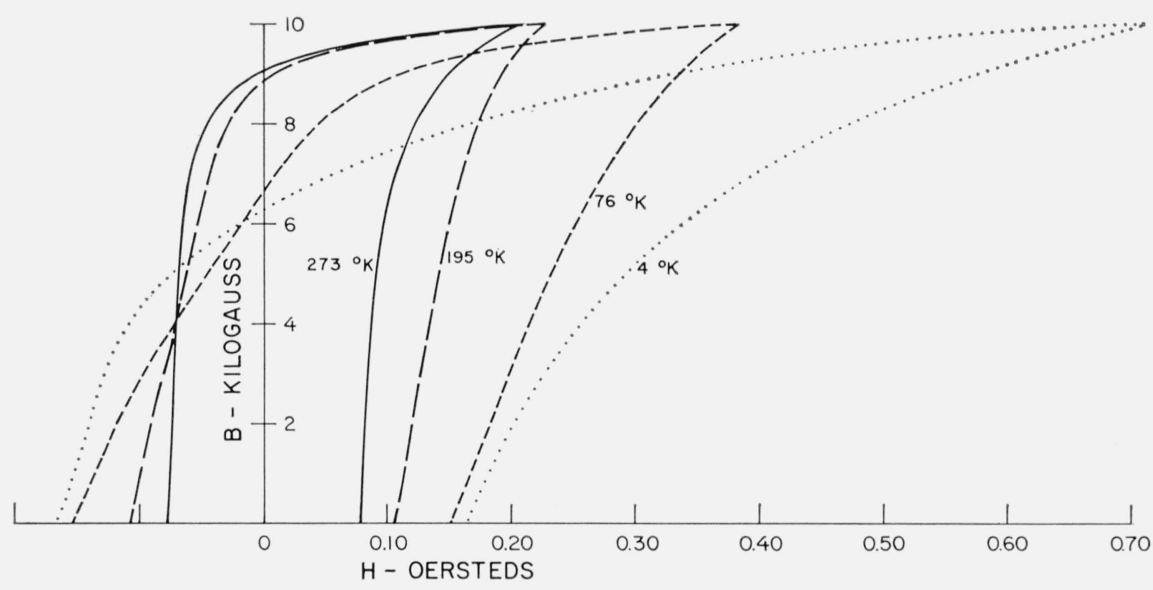

Figure 19. Hysteresis loops. Material 211.

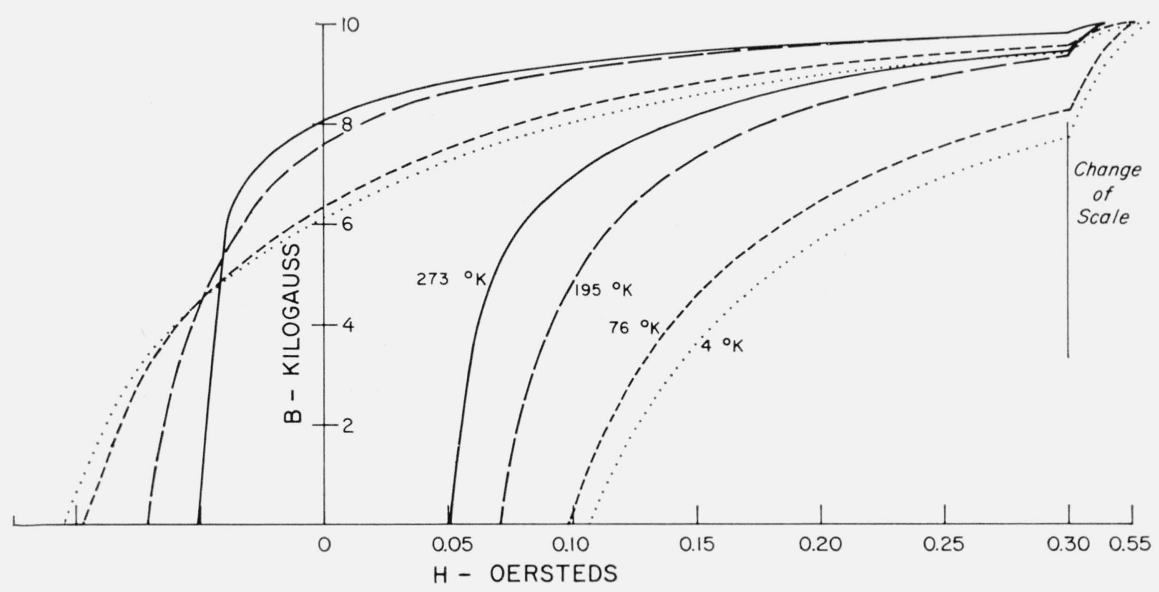

Figure 20. Hysteresis loops.

Material 213. 
general more temperature sensitive than the $\mathrm{Fe}-\mathrm{Si}$ alloys. Hysteresis loss increases on the order of 100 percent have been measured for some materials at $4^{\circ} \mathrm{K}$. The large decrease in residual induction at low temperatures may make this material less desirable for certain applications.

The authors gratefully acknowledge the assistance of the following people during the course of this project: W. M. Lightner of United States Steel Corporation and D. C. Dieterly of Armco Steel Corporation for supplying the material, D. O. Schwennesen of Magnetic Metals Company and C. E. Herr of Westinghouse Electric Corporation for fabrication of the samples, R. L. Powell and D. MacDonald for initiating much of the early project planning, J. C. Moulder for taking some of the experimental measurements, G. H. Wallace for equipment preparation, and N. V. Frederick and R. D. Harrington for use of the B-H loop tracer.

\section{References}

[1] R. L. Sanford and I. L. Cooter, Basic magnetic quantities and the measurement of the magnetic properties of materials, National Bureau of Standards Monograph 47, (1962).

[2] ASTM Standards 1964, Part 8, A 343-60T, Alternating current magnetic properties of materials using Epstein specimens, ASTM, Philadelphia (1964).

[3] C. W. Chen, Temperature dependence of magnetic properties of silicon-iron, J. Appl. Phys. 29, 1337 (1958).

[4] E. Tatsumoto and T. Okamoto, Temperature dependence of the magnetostriction constants in iron and silicon iron, J. Phys. Soc. Japan 14, 1588-94 (1959).

[5] K. B. Vlasov and Ya. S. Shur, Temperature dependence of the coercive force in monocrystals of transformer steel, in Russian, J. Tech. Phys., USSR 21, 39-50 (1951). 